\title{
La Cooperación de los Ejércitos Argentino y Brasileño desde Fines del Siglo XX*
}

\section{The Cooperation of the Argentine and Brazilian Armies Since the End of the 20th Century}

Rev. Bra. Est. Def. v. 2, no 1, jan./jun. 2015, p. 154 - 167

ISSN 2358-3932

\author{
GUILLERMO LAFFERRIERE*** \\ GERMÁN SOPRANO****
}

\section{1. iNTRODUÇÃO}

Desde mediados de la década de 1980 Argentina y Brasil avanzaron en acuerdos que redundaron en la constitución del Mercado Común del Sur (MERCOSUR). El estudio de los conflictivos y no lineales procesos de cooperación e integración que resultaron de aquellos acuerdos ha sido comprendido principalmente en sus dimensiones económica y política y, en menor medida, educativa y cultural. Menos visible para el debate público o como objeto de investigación académico ha sido su correlato en el diseño y desarrollo de medidas de confianza mutua y cooperación en defensa nacional y seguridad internacional, más precisamente, en relación con los intercambios y experiencias compartidas por sus Fuerzas Armadas que, durante casi todo el siglo XX, mantuvieron hipótesis de conflicto vecinales que incidieron en sus doctrinas y educación, organización, dimensiones y despliegue militar, así como en su equipamiento y logística.

Este trabajo tiene como objetivo analizar ejercicios combinados del Ejército Argentino y el Ejército Brasileño desde fines del siglo XX. Nos proponemos: (1) Identificar políticas que orientaron su concepción y con-

\footnotetext{
* Este trabajo es parte de una investigación de la Universidad Nacional de Quilmes, con dirección de Germán Soprano: "Política de defensa y reestructuración de las Fuerzas Armadas Argentinas entre fines del siglo XX y principios del XXI. Cambios, continuidades y desafíos".

** Profesor de la Universidad J.F. Kennedy (Argentina). e-mail: glafferriere@yahoo.com.ar *** Investigador del CONICET / Profesor de la Universidad Nacional de Quilmes y Universidad Nacional de La Plata (Argentina). e-mail: gsoprano69@gmail.com.
} 
creción. (2) Destacar que en una primera etapa se produjeron ejercicios clásicos o convencionales contra un enemigo estatal que controla un espacio geográfico y, posteriormente, se incorporaron operaciones de apoyo a la comunidad o de asistencia en situaciones de catástrofes. ${ }^{1}$

Sostendremos que desde la década de 1990 la política de defensa argentina se ha manifestado en favor de la construcción de una integración regional en el Mercosur y, más recientemente, en la UNASUR; mientras que la política del Brasil ha promovido la cooperación regional en defensa, procurando con ello preservar una mayor autonomía. Pero esa vocación argentina por la integración ha sido inconstante en sus realizaciones prácticas. En tanto que la más limitada cooperación del Brasil, por el contrario, sostuvo los compromisos asumidos, al menos, en relación con los ejercicios efectuados con el Ejército Argentino.

\section{UNA NUEVA RELACIÓN ENTRE ARGENTINA Y BRASIL EN MATERIA DE DEFENSA NACIONAL Y SEGURIDAD INTERNACIONAL EN DEMOCRACIA}

Las relaciones entre las Fuerzas Armadas de la Argentina y el Brasil, particularmente de sus componentes terrestres, cambiaron sensiblemente desde que se concretó un cambio histórico en las relaciones bilaterales con los acercamientos promovidos por sus gobiernos civiles electos en forma democrática al promediar la década de 1980. No obstante, ya en la década de 1970 se habían manifestado señales de cambio cuando se resolvieron disputas sobre el empleo de recursos hídricos para su aprovechamiento en la generación de energía eléctrica. Antes de ese período ambos países mantuvieron hipótesis de conflicto en torno del control geopolítico de la Cuenca del Plata y, más ampliamente, por el liderazgo subregional en el Cono Sur y Sudamérica. La conflictividad en la relación con el Brasil en el caso argentino llegó al extremo de postergar el desarrollo de la región mesopotámica para emplear el Río Uruguay y principalmente el Río Paraná como obstáculos naturales donde apoyar su defensa ante una potencial acción ofensiva brasileña.

La Argentina fue definiendo desde entonces una política de defensa de carácter defensiva, autónoma y cooperativa. La misión principal de sus Fuerzas Armadas consistió -según el decreto 1.691 de 2006 de Directiva sobre Organización y Funcionamiento de las Fuerzas Armadas - en "conjurar y repeler toda agresión externa militar estatal”; y sus "misiones subsidiarias" cuatro: participar en el marco de operaciones multilaterales de Naciones 
Unidas, de seguridad interior previstas por la Ley 24.059 de Seguridad Interior, de apoyo a la comunidad nacional o de países amigos, y en la construcción de un sistema de defensa subregional. Con relación a esta última se ha sostenido que el diseño del instrumento militar debe garantizar la interoperabilidad y complementariedad con Fuerzas Armadas del MERCOSUR o de UNASUR. No obstante, este posicionamiento argentino no ha tenido a comienzos del siglo XXI una adecuada correspondencia con sus más bien magros esfuerzos políticos e inversión en recursos humanos, materiales y financieros destinados a concretarlos; tal como se constató en 2013 con la ausencia de su Fuerza Aérea en el ejercicio combinado aéreo CRUZEX en el nordeste del Brasil, o bien con el creciente recurso a ejercicios combinados de gabinete ante las restricciones presupuestarias para solventar gastos de traslado al terreno de personal, vehículos y equipamiento del Ejército.

Por su parte, el Brasil definió su política de defensa en democracia a partir de una pluralidad de funciones que asignó a sus Fuerzas Armadas en la Constitución Federal del Brasil de 1988. Particularmente su artículo 142 estableció como misiones la "defensa de la Patria” contra agresiones militares exteriores, la "garantía de los poderes constitucionales y, por iniciativa de cualquiera de estos, de la ley del orden”. De acuerdo con el Livro Branco da Defesa Nacional de 2012 su política de defensa tiene por objetivos: Garantizar la soberanía, el patrimonio nacional y la integridad territorial. Defender los intereses nacionales y las personas, los bienes y los recursos brasileños en el exterior. Contribuir a la preservación de la cohesión y unidad nacionales; a la estabilidad regional; y el mantenimiento de la paz y seguridad internacionales. Intensificar la proyección del Brasil en el concierto de las nacionales y su mayor inserción en los procesos decisorios internacionales. Mantener las Fuerzas Armadas modernas, integradas, adiestradas y balanceadas, y con creciente profesionalización, operando de forma conjunta y adecuadamente desplegadas en el territorio (Ministério da Defesa do Brasil, 2012: 24).

A diferencia del caso argentino, no hay en estos enunciados programáticos brasileños invocaciones explícitas a favor de la integración regional. Esta última diferencia puede explicarse - como ha señalado Samuel Alves Soares - en virtud de la configuración de culturas estratégicas divergentes entre la Argentina y el Brasil. Al respecto en otro trabajo (Soares; Soprano, 2014) sostuvimos que en materia de cooperación en defensa regional en América del Sur, los gobiernos de Néstor Kirchner y Cristina Fernández de Kirchner han manifestado públicamente su compromiso con una políti- 
ca de integración regional.

En tanto los gobiernos de Luiz Inácio Lula da Silva y Dilma Rousseff han destacado más bien la dimensión cooperativa de la defensa regional, posiblemente debido a que la inversión de esfuerzos procurando un consenso en torno de una política de defensa suramericana obraría para políticos, diplomáticos y militares del Brasil - país que aspira a un asiento permanente en el Consejo de Seguridad de la ONU sin el apoyo de sus vecinos - más como un lastre que como un factor potenciador de su proyección global. Dicho esto debe insistirse una vez más que la búsqueda de la integración regional en defensa expresada por esos gobiernos argentinos ha sido más bien un posicionamiento inconsecuente y en el que han gravitado también sus serios déficits en materia de defensa; al tiempo que el más limitado objetivo cooperativo brasileño ha contado hasta ahora con muestras más efectivas de concreción.

\section{EJERCICIOS COMBINADOS DE LOS EJÉRCITOS DE ARGENTINA Y BRASIL}

Como señalábamos al comienzo de este trabajo, con el fin de los gobiernos autoritarios de los dos países en la década de 1980, sus Ejércitos se encontraban en condiciones de construir unas nuevas relaciones bilaterales, con renovados intercambios de experiencias y con vistas a avanzar en un proceso de cooperación e integración, que requería solamente de la decisión política para que ello fuera posible. ${ }^{2}$

Los ejercicios combinados no fueron objeto de atención específica en la redacción del Libro Blanco de la Defensa Argentina de 1999. En sus páginas se bregaba en favor de la cooperación e integración en el MERCOSUR, Sudamérica y Latinoamérica, se invocaba la cooperación en materia de seguridad internacional en el marco de la Organización de Estados Americanos, pero no se formulaban definiciones sobre ejercicios con Fuerzas Armadas de países de la región. Esta indefinición fue revertida en el Libro Blanco de la Defensa Argentina de 2010, determinándose que:

[l]as ejercitaciones combinadas hacen a la propio definición política de la Defensa Nacional en tanto contribuyen al incremento y consolidación de la confianza mutua entre los Estados y son fundamentales para la construcción de un sistema de defensa subregional, coadyuvando asimismo al fortalecimiento de la región suramericana como `zona de paz'. Además, desde la dimensión técnico-militar, las ejercitaciones combinadas incrementan y comprueban los grados de interoperabilidad del instrumento militar con terceras naciones, los que son regularmente puestos en práctica en las misiones de paz 
RBED, v. 2, no 1, jan./jun. 2015

de las Naciones Unidas (Ministerio de Defensa Argentina, 2010: 180).

Asimismo en 2010 se fijaron criterios para priorizar el diseño y concreción de ejercicios combinados bilaterales y multilaterales tales como:

La promoción y afianzamiento de la confianza mutua y la profundización de los niveles de interoperabilidad militar con las otras FFAA del ámbito regional y subregional.

El desarrollo y consolidación de aptitudes relacionadas con la misión principal del instrumento militar y con la participación en ejercitaciones multilaterales en el marco del sistema de seguridad colectiva (operaciones de paz en sus diversas modalidades), y el perfeccionamiento en las operaciones de apoyo a la comunidad nacional o de países amigos en situaciones de catástrofe o desastres naturales.

El incremento concreto y comprobación de los niveles de capacidad propios de los grados de interoperabilidad militar con las naciones del ámbito regional y del subregional. En la misma línea, la incorporación de códigos y procedimientos operacionales de creación reciente y el impulso al desarrollo, modernización y consolidación de la doctrina operacional combinada con dichos países.

La optimización de las capacidades necesarias para las actividades combinadas que involucran planeamiento, conducción y ejecución de las operaciones en el terreno.

La obtención de parámetros concretos para el diseño y determinación de las capacidades del instrumento militar. Parámetros que refieran el logro y la consolidación de los estándares mínimos de interoperabilidad para una deficiente interacción militar de carácter ampliado y multilateral.

La consolidación de códigos y procedimientos tendientes a la promoción de mayores niveles de entendimiento y coordinación ante crisis o contingencias (Ministerio de Defensa Argentina, 2010: 181).

En tanto que se estableció una distinción entre dos tipos de adiestramiento en ejercicios combinados. Por un lado, las denominadas operaciones militares propiamente dichas (cuyo contenido no se explicitaba, si bien se trataba de ejercicios contra un enemigo estatal externo) y, por otro, operaciones de paz o de asistencia en catástrofes y emergencias humanitarias.

Por su parte, el Livro Branco da Defesa Nacional de Brasil, publicado por primera vez en 2012, no fijaba ningún posicionamiento específico en relación con los ejercicios combinados con Fuerzas Armadas de otros países, si bien plantea su compromiso con la paz internacional, la promoción de la multipolaridad cooperativa, y la cooperación en América del Sur en el marco de la UNASUR y el CDS con el objetivo de contribuir a una comunidad de seguridad con los países vecinos con los cuales - sostenía-se comparten "experiencias históricas comunes, desafíos de desarrollo 
semejantes y regímenes democráticas, que facilitan la comprensión recíproca y propician una acomodación pacífica de los diversos intereses nacionales" (Ministério da Defesa do Brasil, 2012: 29 nuestra traducción).

Inicialmente, entonces, en marco del establecimiento de medidas de confianza mutua y cooperación en el MERCOSUR se fueron desarrollando ejercitaciones entre Argentina y Brasil que sorprendentemente adquirieron una continuidad y creciente complejidad, incorporando también en algunas oportunidades a los Ejércitos de otros países del MERCOSUR. Entre ellas podemos mencionar: ${ }^{3}$

- En 1993 un ejercicio combinado de planeamiento entre Argentina, Brasil y Uruguay realizado en la Brigada Escuela del Ejército Argentino en la ciudad de Curuzú Cuatiá (provincia de Corrientes) y en 1994 el ejercicio de planeamiento de operaciones tácticas Ejércitos Amigos en la Escuela Superior de Guerra del Ejército en la ciudad de Buenos Aires con participación de Argentina, Brasil, Paraguay y Bolivia e incorporación de Chile y Perú como observadores.

- En 1996 se desarrolló el ejercicio Cruz del Sur entre los Ejércitos de Argentina y Brasil - con participación de Paraguay como observador - sobre la base del planeamiento de Operaciones de Paz, integrando elementos de asesoramiento (Estados Mayores) de nivel Brigada y aún de nivel unidades de combate, comprendiendo en una situación figurada la planificación completa de una operación en territorio argentino (Provincia de Corrientes).

- En 1997 Uruguay se integró al ejercicio Cruz del Sur y Paraguay y Chile participaron como observadores. La ejercitación en territorio del Brasil (Estado de Rio Grande do Sul) contó con el despliegue de tropas en el terreno. Debe destacarse como un hecho inédito en el siglo XX que con estos ejercicios Cruz del Sur el Ejército Argentino se desplegó en territorio brasileño y otro tanto ocurrió con el Ejército Brasileño en la Argentina.

- El ejercicio Cruz del Sur se efectuó en 1998 en territorio argentino y en 1999 en el Brasil, contando desde este último año con la participación de Uruguay y Paraguay.

- En 1999 se hizo el ejercicio Operación Iguazú entre Argentina y Brasil en la ciudad de Posadas (Provincia de Misiones) afrontando una situación de catástrofe natural por inundación. 
RBED, v. 2, no 1 , jan./jun. 2015

- En 2000 el ejercicio Cruz del Sur se efectuó por última vez, realizándose en Rosario do Sul (Estado do Rio Grande do Sul) e incluyó a otros países del MERCOSUR.

- En 2001 el ejercicio Iguazú II en Foz do Iguaçu (Estado de Paraná) comprendió la planificación de acciones de apoyo a organismos de defensa civil ante desastres naturales - con la participación de Bolivia como observador.

- En 2002 se concretó por primera vez el ejercicio Lazo Fuerte en la ciudad de Paraná (Provincia de Entre Ríos), consistiendo en la formación de un comando combinado argentino-brasileño para el planeamiento de una operación militar convencional.

- En 2004 se efectuó el Lazo Fuerte como ejercicio combinado de gabinete de operaciones convencionales en la ciudad de Santa María (Estado do Rio Grande do Sul); y se sustanciaron con unidades de paracaidistas los ejercicios Duende - de operaciones convencionales - en Córdoba (Provincia de Córdoba) y el SACI - también de operaciones convencionales - en Rosario do Sul (Estado do Rio Grande do Sul).

- En 2005 se realizaron en la ciudad de Paraná (Provincia de Entre Ríos) y 2006 en el noroeste argentino los ejercicios combinados convencionales Operación Hermandad, concebidos como adiestramiento de Estado Mayor y ejecutados en fases de planeamiento y simulación de combate. El primero contó con la participación de Chile como observador y el segundo con participación de efectivos de Argentina, Brasil, Paraguay, Uruguay, Bolivia y Venezuela.

Presentamos a continuación un cuadro con ejercicios bilaterales de los Ejércitos de la Argentina y el Brasil entre 2007 y 2013. Nótese que se han diversificado en cantidad, en diferentes ambientes geográficos y en niveles de complejidad técnica importantes. 
Cuadro $\mathrm{N}^{\mathrm{o}}$. Ejercicios combinados de Ejércitos de Argentina y Brasil entre 2007-2013 ${ }^{4}$

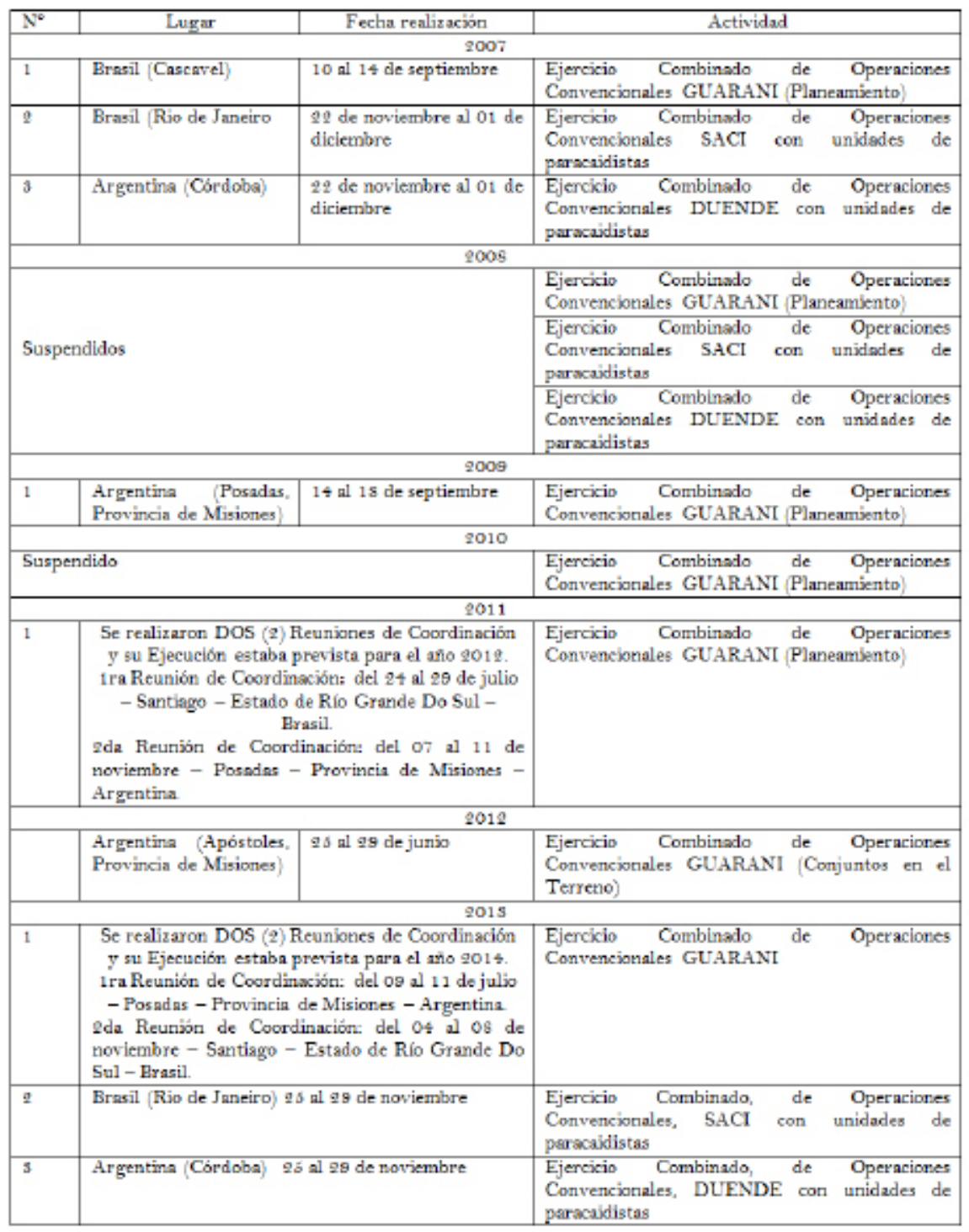

El cuadro $\mathrm{N}^{\mathrm{o}} 1$ es ilustrativo de la continuidad que han tenido las ejercitaciones militares bilaterales con Brasil desde que comenzaron a llevarse a cabo a mediados de la década de 1990. Las ejercitaciones 
cambiaron de denominación - Guarani, Duende, Saci - en la medida que fueron sumándose actividades nuevas, aunque todas siguieron el patrón que describiremos más adelante en relación con el Lazo Fuerte. Es particularmente notable que se haya mantenido el foco de atención en las ejercitaciones de tipo convencional, es decir en aquellas que tienen por objeto adiestrar en las operaciones contra fuerzas enemigas regulares, aún cuando en un comienzo el Cruz del Sur otorgó especial hincapié en operaciones propias de las tropas de mantenimiento de paz como en la asistencia a la población en situaciones de catástrofe. ${ }^{5}$ En contrapartida, también cabe destacar que en 2008 fueron suspendidos los ejercicios Duende, Saci y Guarani y en 2010 el Guarani, incluso cuando al menos en dos casos se trataba de ejercicios de planeamiento que requerían de una presencia muy menor de efectivos y no tenían necesidad de contar con tropas en el terreno que concretaran el planeamiento previo. En ambas oportunidades la suspensión pudo ser ocasionada por restricciones económicas de la Argentina. Cualquiera haya sido la causa de la suspensión, ello implicó una discontinuidad para un esfuerzo que venía sosteniéndose con acciones concretas que fortalecían lazos entre los Ejércitos y producían experiencias de interoperabilidad de altísimo valor militar.

Debe asimismo mencionarse que los ejercicios combinados ArgentinaBrasil se llevaron a cabo en el marco de una cada vez más creciente diferencia tecnológica, presupuestaria y hasta administrativa entre ambos Ejércitos. El componente terrestre de las Fuerzas Armadas Argentinas no ha iniciado un proceso de modernización concreto que permita una renovación profunda de sus medios y estructuras organizativas que los contienen, ya sea porque éste no ha sido un objetivo prioritario de la política pública de los diferentes gobiernos nacionales ni de la dirigencia política con representación en el Congreso de la Nación en democracia, y/o debido a que las conducciones de las Fuerzas Armadas Argentinas no han formulado o no han podido ofrecer al poder político alternativas para una profunda reestructuración del instrumento militar de la defensa en el siglo XXI. ${ }^{6}$

\section{CONCLUSIONES}

Como decíamos en otro trabajo (Soares; Soprano, 2014), mientras que Argentina ha afirmado desde la década de 1990 la necesidad de una integración en materia de defensa a nivel regional suramericano, el Brasil es 
más bien partidario de una cooperación con vistas a resguardar su autonomía, en virtud de la desigual relación que reconoce con sus vecinos y procurando consolidar su rol como un global player con base en Sudamérica y proyección en el Caribe, el Atlántico Sur, la Antártida, África Occidental y África luso-hablante, y aspirando asimismo a ocupar un puesto permanente en el Consejo de Seguridad de Naciones Unidas. Es por ello que el énfasis otorgado por la Argentina a los ejercicios combinados - al menos desde sus posicionamientos políticos-discursivos, esto es, en un plano que no supone una necesaria correspondencia con los esfuerzos políticos-institucionales e inversión efectiva de recursos materiales y humanos - guarda relación con su definición de una política de defensa defensiva, autónoma y cooperativa. Dicha opción conjuga esa elección político-ideológica con otra menos prosaica -expresiva de las debilidades estructurales de su sistemapor la cual Argentina termina delegando parcialmente en los otros países de la región las responsabilidades de su propia defensa nacional.

Creemos pues que el presente trabajo permite mostrar la manera en que los Ejércitos de la Argentina y el Brasil avanzaron en actividades operacionales concretas en el marco de una circunstancia política favorable a la cooperación en defensa. Como ocurre en la interacción bilateral entre Estados, no es extraño que se den esos avances parciales entre estructuras organizativas aun cuando en otros campos existan conflictos que dificultan aspectos como los económicos que - prima facie - aparecen como más relevantes. Pero al mismo tiempo, el proceso de avance evidenciado por los ejercicios combinados convencionales tiene límites en la incapacidad de parte de la Argentina de poder reunir elementos tecnológicamente similares para interactuar con el Brasil en el campo práctico de las ejercitaciones comunes. La experiencia positiva adquirida en ejercicios convencionales como Lazo Fuerte constituyó una base para ejercitaciones combinadas como Duende, Saci y Guarani, siendo estos a su vez antecedentes básicos para potenciar nuevos intercambios bilaterales o en el ámbito del Consejo de Defensa Suramericano.

Ahora bien, en los últimos años en la Argentina la dirigencia política viene enfatizando en sus discursos públicos el empleo del recurso militar en relación con sus misiones subsidiarias, esto es, el complimiento de funciones de desarrollo y atención a la comunidad, en catástrofes naturales o situaciones causadas por factores antrópicos, e incluso de apoyo a las fuerzas de seguridad en asuntos de seguridad interior. Este empleo del recurso militar, que tiene un fundamento lógico en otras consideraciones 
de carácter político interno, ocupa un lugar importante dentro del espectro de posibles usos de la fuerza militar, pero también tiene por consecuencia un aspecto que resulta altamente negativo. Un excesivo acento en ese tipo de empleos atenta contra el demandante esfuerzo de preparar ese instrumento militar para el cumplimiento de sus funciones convencionales contra agresiones de un enemigo estatal externo. De modo que, en el curso de la próxima década habrá que comprender cuál será la evolución de los ejercicios combinados de los que participen el Ejército Argentino y el Ejército Brasileño, especialmente, analizar cuál será el perfil que la Argentina imprimirá a los mismos, cuáles los recursos y equipamiento implicados y, en definitiva, cuál será su contribución en el proceso de cooperación y/o integración bilateral y regional en materia de defensa y seguridad internacional.

Asimismo, si el Consejo Suramericano de Defensa, además, privilegiara las actividades no directamente relacionadas con aspectos específicamente militares en desmedro de la cooperación a partir de ejercicios combinados convencionales, entonces se estarán dirigiendo los recursos militares regionales disponibles en la concreción de capacidades muy alejadas de las esperables de un Consejo que tiene a la defensa como su centro. Proceder de esta manera hará que en el futuro nos cuestionemos acerca de si el Consejo no correrá la suerte de otras iniciativas del pasado que, mas allá de crear estructuras político-administrativas, se volvieron incapaces de avanzar en hechos concretos en pos de las metas que llevaron a su creación. O quizás debemos plantearnos si dentro del sistema creado en esa organización habrá Estados que retengan capacidades efectivas para actuar ante contingencias que requieran el empleo de fuerzas militares convencionales, y otras que sólo aportarán fuerzas para contingencias provenientes de desastres naturales o causados por el hombre o en misiones de apoyo a la comunidad y desarrollo. Si éste último fuera el caso, cabría preguntarnos dónde quedó el cuestionamiento que diversos actores gubernamentales, políticos, militares y académicos de los países suramericanos han efectuado a la visión que predominantemente se promueve desde Estados Unidos a favor del empleo de las Fuerzas Armadas de la región en torno de las denominadas nuevas amenazas. 


\section{NOTAS}

1. Considerando los límites impuestos a la extensión del artículo, no describiremos aquí el diseño del instrumento militar terrestre de la Argentina y el Brasil, particularmente, en lo referente a la configuración de la Primera División del Ejército Argentino emplazada en la región nordeste del país, y el Comando Militar Sur del Ejército Brasileño. Para un análisis de esas configuraciones remitimos a Lafferriere y Soprano (2014).

2. Sérgio Aguilar (2010) recuerda que mientras que los marinos acumularon experiencias en ejercicios combinados - principalmente en coordinación con los Estados Unidos- durante la Guerra Fría, los Ejércitos de la región sólo comenzaron a hacerlos en la década de 1990.

3. La información fue reunida y consolidada de diferentes fuentes: http:// www.eb.mil.br/apresentacao.http://www.eb.mil.br/apresentacao.http://www. nuevamayoria.com/ES/INVESTIGACIONES/defensa/041222a. Consultados en línea el 16 de junio de 2014.

4. Este cuadro fue compendiado de diferentes fuentes - entre ellas el Atlas de la Red de Seguridad y Defensa de América Latina (Donadío; Tibiletti, 2010) y fuentes oficiales de Argentina que se mantienen en reserva.

5. Estos ejercicios combinados convencionales pueden, no obstante, incluir algún tipo de actividad complementaria de apoyo a la comunidad, tal como fuera consignado por un medio de prensa de la Provincia de Misiones en ocasión de la realización del Guarani de 2012. Entonces algunos efectivos realizaron tareas en beneficio de una escuela pública de la localidad de Tacuaruzú. http:// misionesparatodos.com/ejercicio-del-ejercito-argentino-en-conjunto-consoldados-brasilenos. Consultado en línea el 30 de junio de 2014. 6. A estas dificultades estructurales deben sumarse procedimientos políticoadministrativos como la necesaria autorización del Congreso de la Nación a la salida de fuerzas propias del país e el ingreso de extranjeras, que no siempre se cumplimentan con tiempo suficiente para concretar las actividades programadas en tiempo y forma. 
RBED, v. 2, no 1, jan./jun. 2015

\section{LA COOPERACIÓN DE LOS EJÉRCITOS ARGENTINO Y BRASILEÑO DESDE FINES DEL SIGLO XX}

\section{RESUMEN}

Este trabajo tiene como objetivo analizar ejercicios combinados del Ejército Argentino y el Ejército Brasileño desde fines del siglo XX. Nos proponemos: 1) Identificar políticas que orientaron su concepción y concreción. 2) Mostrar que en una primera etapa se produjeron ejercicios clásicos o convencionales contra un enemigo estatal que controla un espacio geográfico y, posteriormente, se incorporaron operaciones de apoyo a la comunidad o de asistencia en catástrofes. Sostendremos que desde la década de 1990 la política de defensa argentina se ha manifestado en favor de la construcción de una integración regional en el Mercosur y, más recientemente, en la UNASUR; mientras que la política del Brasil ha promovido la cooperación regional en defensa, procurando con ello preservar una mayor autonomía. Pero esa vocación argentina por la integración ha sido inconstante en sus realizaciones prácticas. En tanto que la más limitada cooperación del Brasil, por el contrario, sostuvo los compromisos asumidos, al menos, en relación con los ejercicios efectuados con el Ejército Argentino.

Palabras clave: Ejército. Ejercicios Combinados. Argentina. Brasil.

\section{ABSTRACT}

This work aims to discuss combined exercises of the Argentine and the Brazilian Army since the end of the 20th century. We propose: 1) Identify policies that guided its design and development. 2) Show that occurred in a first stage in classic or conventional exercises against an conventional estatal enemy that controls a geographical space and latter, subsequently, other no war military operations were incorporated to practice civilian population assistance in catastrophes. We think that since the 1990 s the Argentine defence policy has manifested itself in favour to build a regional integration in the level of MERCOSUR and, more recently, in the UNASUR. While the Brazilian policies have promoted regional cooperation in defence, seeking to preserve greater autonomy. But Argentine's commitment to integration has been inconsistent in their practical realizations. In contrast, Brazil has maintained its level of cooperation with the exercises carried forward with the Argentine Army.

Keywords: Army. Combined Exercises. Argentina. Brazil. 


\section{REFERENCIAS}

Aguilar, Sérgio Luiz Cruz. (2010) Segurança e defesa no Cone Sul. Da rivalidade da Guerra Fria à cooperação atual. Porto de Ideias Editora, São Paulo.

Kuhlmann, Paulo. (2007) Exército Brasileiro: estrutura militar e ordenamento político. 1984-2007. Tese de Doutorado. Departamento de Ciencia Política. Faculdade de Filosofia, Letras e Ciências Humanas. Universidade de São Paulo, São Paulo.

Kuhlmann, Paulo. (2012) "Mudança de missoes e estructura de defesa do Exército Brasileiro (1985-2007)”. XI Brazilian Studies Association Congress. Illinois.

Lafferriere, Guillermo; Soprano, Germán. (2014) "Vino nuevo en odres viejos. La política de defensa nacional de la Argentina democrática y la configuración de su instrumento militar”. Revista Brasileira de Estudos Estratégicos, No. 7, Vol. 1.

Instituto de Estudos Estratégicos. Universidade Federal Fluminense, Niteroi (en prensa).

Ministerio de Defensa Argentina. (1999) Libro Blanco de la Defensa Argentina. Ministerio de Defensa, Buenos Aires.

Defensa, Buenos Aires. (2010) Libro Blanco de la Defensa Argentina. Ministerio de

Ministério da Defesa do Brasil. (2012) Livro Branco de Defesa Nacional. Ministério da Defesa, Brasília.

Donadio, Marcela; Tibiletti, Paz (eds.) (2010) Atlas comparativo de la defensa en América Latina y Caribe. Resdal, Buenos Aires.

Soares, Samuel Alves; Kuhlmann, Paulo Loyolla. (2005) "Las relaciones civilesmilitares en Brasil”. In: J. Olmeda (comp.) Democracia frágiles. Las relaciones civiles-militares en el mundo iberoamericano. Tirant Lo Blanch, Valencia, pp: 463522.

Soares, Samuel Alves; Soprano, Germán. (2014) "Políticas de defesa de Argentina e Brasil no começo do século XXI: entre a confiança mútua e as culturas estratégicas em dissonância”. In: Passos, R.D.F; Vieira, N.R.; Simonetti, Mirian C. L. (eds). Relações Internacionais Contemporâneas: novos protagonistas e novas conjunturas. Cultura Acadêmica / Universidade Estadual Paulista / CAPES / Oficina Universitaria. Marília, São Paulo, pp: 187-210.

Svartman, Eduardo Munhoz. (2006) Política externa e região em tempos de crise. Méritos Editora, Passo Fundo.

. (2014) “Da II Guerra Mundial à Guerra Fria. Conexões entre os exércitos do Brasil e dos Estados Unidos”. Latin American Research Review, vol. $49 \mathrm{~N}^{\mathrm{o}}$ 1, pp: 83-103.

Recebido em: 12/09/2014. Aprovado em: 20/02/2015. 